\title{
Behavior of Complex Knots in Single DNA Molecules
}

\author{
Xiaoyan R. Bao, Heun Jin Lee, and Stephen R. Quake \\ Department of Applied Physics, California Institute of Technology, Pasadena, California 91125, USA
}

(Received 1 August 2003; published 31 December 2003)

\begin{abstract}
We used optical tweezers to tie individual DNA molecules in knots. Although these knots become highly localized under tension, they remain surprisingly mobile and undergo thermal diffusion with classical random walk statistics. The diffusion constants of knots with different complexities correlate with theoretical calculations of knot sizes. We show that this correlation can be explained by a simple hydrodynamical model of "self-reptation" of the knot along a polymer.
\end{abstract}

DOI: 10.1103/PhysRevLett.91.265506

PACS numbers: 61.41.+e, 02.10.Kn, 87.15.-v

Knots and braids have fascinated observers since ancient times; complex topological designs have been preserved on tiles and pottery dating back thousands of years [1]. Kelvin and Tait undertook the first systematic study of knots in the 19th century [2,3], and in the 20th century a powerful mathematical framework was constructed to classify knots according to various topological invariants [4]. They appear in many different scientific contexts, ranging from the synthesis of topologically nontrivial molecules in chemistry [5] to the role of topology in life itself: topoisomerase enzymes exist to measure and change the topology of DNA. In physics, it was realized quite some time ago that there is a deep connection between knot invariants and theories of statistical mechanics [6]. Knots also appear naturally in long polymers [7]; they are, in fact, the generic state and can profoundly impact the dynamics of systems in which they appear $[8,9]$. The magnitude of these effects depends strongly on the complexity of the knot [10]. Such polymeric topological constraints arise naturally in cells during DNA replication [11], and knotting, in particular, has been important in elucidating the mechanisms of DNA recombination [12].

Theoretical and numerical studies of knots in long polymers suggest that they are localized: the amount of polymer directly engaged within the knot is vanishingly small compared to the total length of the polymer $[13,14]$. Distributions of knots have previously been synthesized in bulk samples $[15,16]$, but only in relatively short circular DNA molecules; in this limit the knots are neither localized nor tightly constrained, and, indeed, they were purified and characterized based upon their effects on the properties of the polymer as a whole. We sought instead to confine the knot and study it as an isolated object. By mechanically knotting linear pieces of DNA with beads at the ends that act as handles for optical tweezers, we were able to keep the DNA under a fixed tension, making the knot a localized structure whose properties are independent of the length of substrate DNA. The confinement of DNA achievable under these conditions allows comparison to models of polymer dynamics, as well as more specific theoretical results for ideal tight knots.
Mechanical knotting of a molecule is challenging because the first step in the tying process involves making a loop in the DNA which then immediately relaxes due to entropic elasticity. Previous experiments tying the simplest possible knot in our lab [J.-C. Meiners (unpublished results)] and elsewhere [17] used non-Newtonian fluids to inhibit this relaxation, but with the unfortunate drawback of suppressing knot dynamics as well. We have now constructed a system capable of tying several different types of knots into DNA molecules and observing their dynamics within a linearly viscous solution. This is therefore the first quantitative and systematic investigation of single, thermally driven polymer knots.

To study how higher-order knotting structure affects dynamics, we constructed knots following the ordering scheme of a standard knot table. Knots are designated by $C_{k}$, with $C$ the minimal number of self-crossings displayed when the knot is projected into a plane, and $k$ a cardinal index used to distinguish between topologically different knots with the same $C$. By threading the DNA loop multiple times, or making more complicated loops before threading them, we were able to consistently construct knots of types $4_{1}, 5_{1}, 5_{2}$, and $7_{1}$, in addition to the simplest knot $3_{1}$ [Fig. 1(d); see also supplemental video [18]]. Approximately 100 different DNA molecules were successfully tied into knots, and about one-third of the knots provided useful quantitative data.

To make bead-DNA-bead dumbbells, $\lambda$ genomic DNA was concatenated with T4 DNA ligase, labeled with biotin at both ends [19], and then mixed with streptavidincoated polystyrene beads ( $1 \mu \mathrm{m}$ diameter, Bangs Labs); dumbbell lengths typically ranged from 40 to $100 \mu \mathrm{m}$. The dumbbells were stained in a solution containing $10 \mathrm{mM}$ Tris- $\mathrm{HCl}(p \mathrm{H} 8), 10 \mathrm{mM} \mathrm{NaCl}, 1 \mathrm{mM}$ EDTA, $6 \%(\mathrm{w} / \mathrm{v})$ poly(ethylene glycol) (PEG, molecular weight 35000 , Polysciences), $250 \mu \mathrm{g} / \mathrm{ml} \alpha$-casein, $0.1 \%$ tween20, and $20 \mathrm{nM}$ YOYO-1 [20]. To reduce photodamage, stained dumbbells were gently mixed with oxygen scavenger solution (equal volume mix of $113 \mathrm{mg} / \mathrm{ml}$ glucose solution, $50 \%$ (v/v) $\beta$-mercaptoethanol, $5 \mathrm{mg} / \mathrm{ml}$ glucose oxidase, and $0.9 \mathrm{mg} / \mathrm{ml}$ catalase) in a $4: 1$ volume ratio just before observation. 


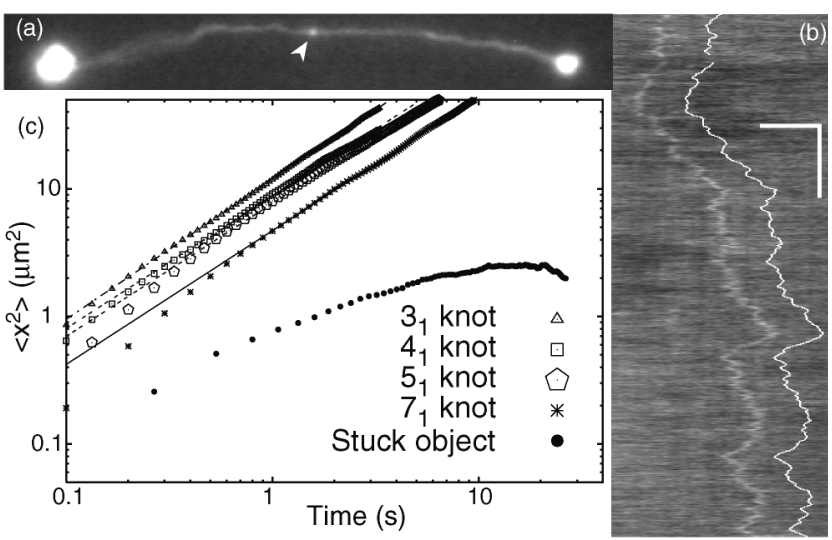

(d)

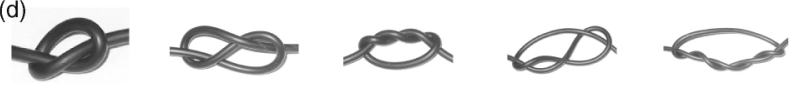

FIG. 1. Observing knot diffusion. (a) The stained, knotted DNA appears as a diffraction-limited contour between two beads, with an increase in fluorescence at the knot (arrow). The path of the DNA is found by software and its intensity profile computed; tiling these profiles from sequential frames (b) reveals a diffusive trace which indicates the knot's trajectory. Scale bars, $5 \mu \mathrm{m}$ (horizontal) and $5 \mathrm{~s}$ (vertical); scale of (a) is the same as the horizontal scale of (b), The trace is quantified (offset white trace) and its mean squared traveled distance as a function of time $t$ computed (c) which obeys a power law with exponent $\alpha \approx 1.06$ (see text). (d) We have tied and analyzed open knots of types (from left to right) $3_{1}, 4_{1}, 5_{1}$, $5_{2}$, and $7_{1}[18]$.

Knotting experiments were performed on a custom inverted optical trapping microscope described previously [21], with the addition of a mercury arc lamp (Oriel) for fluorescence excitation and an image-intensified charge coupled device for detection. A collimating lens in the trapping beam path before the objective was placed on a translation stage driven by a servo motor. Movement of the lens gave depth control of the trap while $x y$ translation was performed by moving the stage supporting the observation flow cell. Each dumbbell was first stretched in an applied flow to give a length calibration, then tied into a knot and observed with the two ends held a fixed distance apart. The type of knot tied was verified during analysis by reviewing the video of the tying process.

All of the knots shrank under tension, becoming highly localized, diffraction-limited spots at tensions as low as $0.1 \mathrm{pN}$ [Fig. 1(a)]. DNA shapes were traced using an active contour ("snake") algorithm [22], and intensity profiles were computed from those shapes. When we tiled intensity profiles from successive frames, bright tracks became identifiable, indicating that the knots move over time [Fig. 1(b)]. In control molecules in which we did not tie a knot, these bright tracks were present about $10 \%$ of the time, close to the expected probability of random thermal knotting in DNA. We quantified these tracks and computed the mean squared traveled distance as a function of time. Logarithmic plots [Fig. 1(c)] in- dicate a power law relation $\left\langle x^{2}\right\rangle \propto t^{\alpha}$. The value of $\alpha$ averaged over all knots was $1.06 \pm 0.02$, in agreement with classical random walk statistics. We then computed diffusion constants by plotting the diffusion data on linear axes and fitting to a line, leaving the offset term free to compensate for short-time artifacts from both observation and analysis; slopes obtained from these fits then become our diffusion constant measurements. We found no discernible tension dependence in knot properties at the tensions $(0.1-2 \mathrm{pN})$ used to extend the DNA molecules. However, the diffusion constant varies dramatically with knot complexity (Fig. 2).

Since the knots have conserved topologies, they must move with a snakelike motion of the polymer through the knot structure, analogous to the reptating motion of a polymer in the presence of a fixed obstacle network. We call this motion "self-reptation" since the same molecule is both constraining and constrained. One can thus define a characteristic time $\tau_{\text {rept }} \sim \frac{L^{2}}{D}$ equal to the time it takes the knot to diffuse through the length $L$ of DNA composing the knot; this time is analogous to the lifetime of the reptation tube. The total length $L$ of polymer engaged within a given knot type has been calculated in numerical

(a)

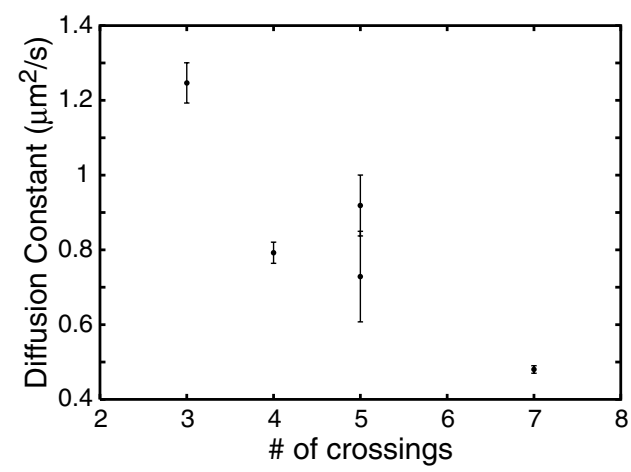

(b)

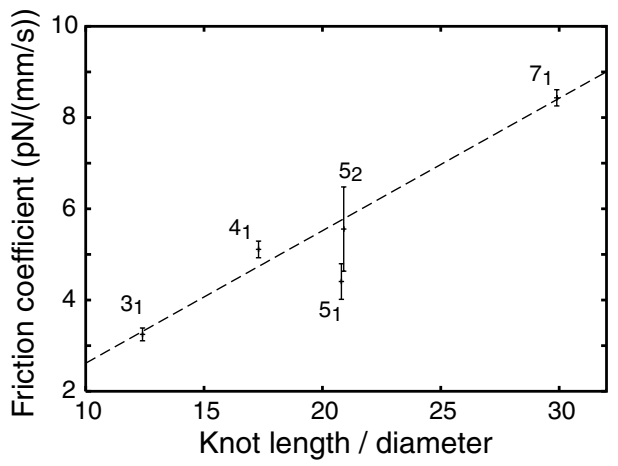

FIG. 2. Knot diffusion constants $D$ vary dramatically with complexity (a); their respective friction coefficients $\zeta=$ $k_{B} T / D$ correlate well to theoretical knot lengths (b). The slope of the best fit line is $0.29 \pm 0.003 \mathrm{pN} /(\mathrm{mm} / \mathrm{s})=(2.9 \pm 0.3) \times$ $10^{-10} \mathrm{~N} /(\mathrm{m} / \mathrm{s})$. Only data points with relative extensions between 0.55 and 0.75 were used. The numbers of knots observed were $23,9,3,4$, and 3 for knot types $3_{1}, 4_{1}, 5_{1}, 5_{2}$, and $7_{1}$, respectively. 
simulations of tight, open knots constructed of ideal ropes [23]. Comparing our drag coefficients $\zeta=\frac{k_{B} T}{D}$ to these lengths yields a roughly linear relationship [Fig. 2(b)], so $D \sim L^{-1}$ and hence $\tau_{\text {rept }} \sim L^{3}$, consistent with the reptation model $[24,25]$ and with observed behavior of DNA in an entangled solution [26].

Besides analyzing the kinetics of knot motion, it is also possible to extract information about the relative sizes of the knots from the microscope images. Using the knot position information from track quantification, we averaged knot intensities and compared them to intensities on adjacent unknotted parts of the DNA. Because the knot sizes are below the resolution of the imaging system, we could measure only the extra amount of DNA present in the knot as compared with an unknotted strand spanning the same distance; this will in general be different from the total knot length $L$ but instead correspond to the change in length caused by the knot in a tight, ideal rope. These dimensionless theoretical lengths $\Lambda_{O}$ are relative to rope diameter and have been studied computationally [23]. Comparing them to the absolute knot length measurements (Fig. 3) gives an average interstrand spacing of $26 \pm 6 \mathrm{~nm}$. This is most likely due to electrostatic self-repulsion of the charged DNA molecule, and the effect is similar in magnitude to computations and observations for DNA in bulk experiments [15].

The above analyses assume that the knot conformations can be approximated as those of ideal tight knots, i.e., conformations which maximize strand-strand distance. Another possibility would be that the knot size is determined by DNA bending rigidity. In that case, topological crossings in the trefoil $\left(3_{1}\right)$ knot would occur along a small part of the loop (as in Fig. 3c of [17]). We can thus calculate the radius of the rigidity-dominant

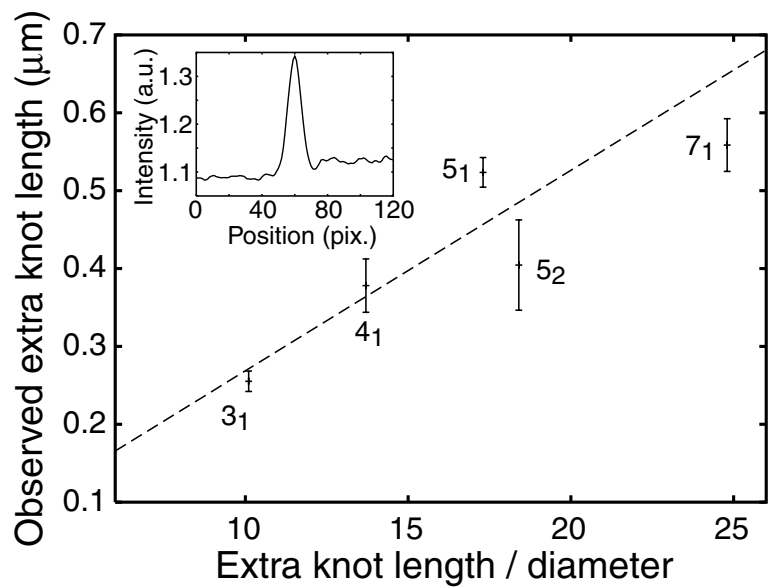

FIG. 3. Averaged extra knot lengths correlate with those determined from numerical simulations of ideal open knots [23]; the slope of the best fit line gives a DNA effective diameter of $26 \pm 6 \mathrm{~nm}$. The ordinate axis represents the difference between the lengths required for a knot and an unknotted segment. knot using elastic bending theory:

$$
R=\sqrt{\frac{k_{B} T \xi_{p}}{2 F}} \simeq \frac{10 \mathrm{~nm} \cdot \sqrt{\mathrm{pN}}}{\sqrt{F}},
$$

where $F$ is the tension on the rope, $k_{B} T$ the thermal energy, and $\xi_{p}$ the persistence length. Since most measurements were performed at $F \sim 1 \mathrm{pN}$, elastic return forces should give an extra length of $2 \pi R \sim 61 \mathrm{~nm}$, or only about one quarter of the $255 \mathrm{~nm}$ trefoil knot size. This argument should be valid for all torus knots, including the $5_{1}$ and $7_{1}$, so it predicts that the sizes of rigiditydominant torus knots are independent of knot type; this is inconsistent with the data in Fig. 3.

Having quantified both the size and mobility of knots, we can propose a simple hydrodynamic model to link these measurements. The constraint network for any given part of the knot is formed by other parts of the same knot, so the "walls" of the effective reptation tube move during diffusion. From computed conformations of ideal knots [23], it appears that the constraining strands in the network are generally perpendicular to the constrained strand; we should therefore expect little or no net force in the direction of strand motion from the constraining strands within a reptating knot. We can then model the friction coefficient as arising from the viscous drag of one long cylinder (the constrained DNA strand) within another (the constraining tube). From [27], p. 54, this gives a drag coefficient per unit length $\zeta / L$ as a function of the ratio $\rho$ of radii of the cylinders and the viscosity $\eta$ :

$$
\frac{\zeta}{L}=\frac{2 \pi \eta}{\ln \rho} .
$$

In this case, $L$ is the length of DNA composing the knot. The inner cylinder radius is half of the hydrodynamic diameter of $2.5 \mathrm{~nm}$ for DNA, and the outer cylinder radius is the observed mean separation between strands of $26 \mathrm{~nm}$. This gives an axial force contribution of $\zeta / L \approx 0.017 \pm$ $0.002 \mathrm{Pas}$ which is in fair agreement with the value of $0.011 \pm 0.003 \mathrm{Pas}$ derived from the slope in Fig. 2(b), especially given the simplicity of the present model. Presumably the flow field associated with the motion of DNA through the knot will "bleed" beyond the phantom tube defined by its constraints, reducing the energy dissipation and giving rise to a slightly lower drag than that predicted by our model.

We have thus shown that knots along an extended DNA molecule behave in a manner consistent with predictions for ideal, tight, reptating knots. Despite having dimensions comparable to the persistence length of DNA, the knots are quite mobile and form one of the smallest known models of reptation. They are also a model system for the study of tightly confined DNA which is present in systems as diverse as eukaryotic nucleosomes and packaged virus heads. Finally, visible 
DNA knots of known topology are ideal substrates for studying the enzymology of topoisomerases at the single molecule level.

We thank D. E. Smith, P. Pieranski, M. Wang, and J. Su for helpful discussion, and J.-C. Meiners, K. Matthews, and A. Groisman for help with preliminary experiments. This work was funded in part by a grant from the Packard Foundation; X. R. B. thanks the Hertz Foundation for financial support.

[1] H. Gardner, Art Through the Ages (Harcourt Brace, Fort Worth, 1996).

[2] W. Thompson, Philos. Mag. 34, 15 (1867).

[3] P. G. Tait, Scientific Papers (Cambridge University Press, London, 1898).

[4] V. F. R. Jones, Lect. Notes Math. 1525, 70 (1992).

[5] Molecular Catenanes, Rotaxanes, and Knots, edited by J.-P. Sauvage and C. Dietrich-Buchecker (Wiley, New York, 1999).

[6] Braid Group, Knot Theory, and Statistical Mechanics, edited by C. N. Yang and M. L. Ge (World Scientific, New Jersey, 1989).

[7] D. W. Sumners and S. G. Whittington, J. Phys. A 21, 1689 (1988).

[8] S. R. Quake, Phys. Rev. Lett. 73, 3317 (1994).

[9] P.-G. de Gennes, Macromolecules 17, 703 (1984).

[10] F. B. Dean, A. Stasiak, T. Koller, and N. R. Cozzarelli, J. Biol. Chem. 260, 4975 (1985).

[11] O. Sundin and A. Varshavsky, Cell 25, 659 (1981).

[12] N. J. Crisona, R. Kanaar, T. N. Gonzalez, E. L. Zechiedrich, A. Klippel, and N. R. Cozzarelli, J. Mol. Biol. 243, 437 (1994).

[13] R. Metzler, New J. Phys. 4, 91 (2002).

[14] V. Katritch, W. K. Olson, A. Vologodskii, J. Dubochet, and A. Stasiak, Phys. Rev. E 61, 5545 (2000).
[15] V.V. Rybenkov, N. R. Cozzarelli, and A.V. Vologodskii, Proc. Natl. Acad. Sci. U.S.A. 90, 5307 (1993).

[16] S. Y. Shaw and J. C. Wang, Science 260, 533 (1993).

[17] Y. Arai, R. Yasuda, K. Akashi, Y. Harada, H. Miyata, K. Kinosita, and H. Itoh, Nature (London) 399, 446 (1999).

[18] See EPAPS Document No. E-PRLTAO-91-001348 for video of a knot constructed by threading a DNA loop. A direct link to this document may be found in the online article'sHTML reference section. The document may also be reached via the EPAPS homepage (http:// www.aip.org/pubservs/epaps.html) or from ftp.aip.org in the directory /epaps/. See the EPAPS homepage for more information.

[19] R. M. Zimmermann and E. C. Cox, Nucleic Acids Res. 22, 492 (1994).

[20] The PEG overlap concentration $c^{*}$ is close to $4.4 \%(\mathrm{w} / \mathrm{v})$. Thus, polymer bundling in the knots should be negligible, and nonlinear drag effects should also be small on the time scales $(30 \mathrm{~Hz})$ of our measurements; this was confirmed by measuring diffusion of $100 \mathrm{~nm}$ yellow fluorescent beads (IDC Latex). We corrected for inhomogeneity in fluorescence intensity from the DNA molecule during data analysis; attempts to find sequence-dependent effects were inconclusive.

[21] J.-C. Meiners and S. R. Quake, Phys. Rev. Lett. 82, 2211 (1999).

[22] M. Kass, A. Witkin, and D. Terzopoulos, Int. J. Comput. Vision 1, 321 (1987).

[23] P. Pieranski, S. Przybyl, and A. Stasiak, Eur. Phys. J. E 6, 123 (2001).

[24] P.-G. de Gennes, J. Chem. Phys. 55, 572 (1971).

[25] M. Doi and S.F. Edwards, The Theory of Polymer Dynamics (Clarendon Press, Oxford, U.K., 1986).

[26] D. E. Smith, T. T. Perkins, and S. Chu, Phys. Rev. Lett. 75, 4146 (1995).

[27] L. D. Landau and E. M. Lifshitz, Fluid Mechanics (Butterworth and Heinemeann, Oxford, U.K., 1987). 